Chapter 17

\title{
Control and Prevention of Obesity and Diabetes Type 2 Through Non-Pharmacological Treatments Based on Marine Products
}

\author{
Daniela Seelenfreund, Pilar Durruty, Nicolas Palou, \\ Sergio Lobos and Rodrigo González
}

Additional information is available at the end of the chapter

http://dx.doi.org/10.5772/56427

\section{Introduction}

Obesity is currently considered as the pandemic of the 21st century and is paradigmatic of non-transmissible chronic diseases, such as type 2 diabetes mellitus (DM2), cardiovascular disease and cancer. Thus, due to its extent, obesity is considered a public health problem that is constantly growing and globally affects both the developed world and developing countries (WHO Prevalence of Diabetes, 2010). In the United States of America (USA) over 30\% of the adult population is obese, the numbers in Europe are between 15 and $20 \%$ and in Chile, with a GDP close to \$US 20,000 per year, the National Health Survey (Encuesta Nacional de Salud, ENS) of 2010, showed a total prevalence of obesity of $27.4 \%$, very similar to industrialized countries (ENS, 2010).

All epidemiologic studies show that obesity is greater in subjects with low education and less in individuals with higher education; in Chile, obesity reaches $35 \%$ in the former. Additionally, obesity increases with people's age, reaching its highest level in the group of those between 50 - 64 years. However, it is very important to mention the increase in obesity in children and adolescents observed in Europe, in Chile and practically throughout the world, and which is the main cause of the appearance in recent years of a constantly increasing number of type 2 diabetes cases in these age groups (ENS, 2010).

Obesity should be considered as a heterogeneous, multifactorial disease with a genetic base more than 60 genes involved have been described - however, predisposition to obesity is not higher than $30 \%$ and environmental factors of individuals and populations account for $70 \%$. The highest impact factor in the development of obesity is excess food intake and the quality 
of the diet with high contents of carbohydrates and fats, to which sedentary lifestyles and lack of physical activity are added, both at work and at leisure, a feature common to all populations nowadays.

Among the causes of obesity, mention should be made of aggressive marketing of non-healthy foods, the low price of the so-called "junk food", higher economic income, as well as higher availability of public transportation and of television sets, video games and internet, all of which lead to an increase in sedentary lifestyles at all ages. Obesity per se is an independent risk factor for coronary cardiopathy, whereby it has been included by the American Heart Association as a major risk factor. Two important investigations, the Framingham and the Finnish prospective study, confirm the direct association between obesity and coronary mortality.

Visceral obesity is the main causing factor of metabolic syndrome (MS), which has been defined as a group of metabolic risk factors (arterial hypertension, dyslipidemia, glucose intolerance), that are more than randomly interrelated with each other and which directly promote the development of DM2 and cardiovascular disease (CVD). In the USA, health surveys show a sustained increase in the prevalence of MS; thus, the National Health and Nutrition Examination Survey (NHANES) for 1988 - 94 showed a prevalence of $23.1 \%$, while the same survey in 1999 - 2006 reported a worrying increase reaching 34\%. The Chilean ENS for 2010 reported very similar figures of 35\% (ENS, 2010), while in 2003 it was $22.6 \%$.

Obesity and particularly android or visceral obesity is associated with type 2 diabetes. It should be highlighted that at diagnosis of DM2, $80 \%$ of the subjects are obese. While this is true for diabetes in general, it should be considered that $90-95 \%$ of diabetic patients suffer diabetes type 2 and only 5 to $10 \%$ correspond to diabetes type 1, of autoimmune pathogenesis not related to obesity. In our country, only $1 \%$ are type 1 diabetic patients.

There is a universal consensus in that obesity is the leading pathogenic factor of pre-diabetes and DM2. Obesity is a determinant of insulin resistance (IR) with compensatory hyperinsulinemia; DM2 becomes manifest when a relative deficiency of insulin secretion is produced.

The International Diabetes Federation estimated a world-wide DM2 prevalence rate of $8.3 \%$ for 2011, which will increase to $9.9 \%$ by 2030 . Some populations, such as Pima Indians from the USA or Mexico and people from the Mauritius Islands with rates higher than $20-30 \%$ are exceptions. Prevalence is growing explosively, thus the WHO reported that in 2010 there existed 250,000,000 diabetic patients in the world and most remarkably predicted a figure of $333,000,000$ for 2025 . The same growth is shown in Chilean investigations, with a progressive increase from 5.3\% in 1981 to 6.3 in 2003 and 9.4\% in 2010. The Chilean survey also shows a clear parallel between prevalence of obesity and DM2 according to age groups (ENS, 2010). The same as with obesity, DM2 is present in higher rates in people with less education and socially more vulnerable groups, which confirms the close association with DM2. It also increases with age, reaching figures close to $20 \%$ in elderly subjects.

Mortality for diabetes due to its chronic complications, particularly macrovascular complications and especially coronary death, is among the 10 leading causes of death worldwide, and the seventh cause of death in Chile. Diabetic patients exhibit 8 to 15 time higher frequency of 
cardiovascular disease (CVD) and 2 to 3 time higher cardiovascular disease mortality than the general population. In the last 40 years, mortality for this cause has decreased in the general population, but not in diabetics. In summary, coronary disease is the main cause of death in DM2 and hyperglycemia is a direct mortality risk factor.

From the above the strong association between obesity, metabolic syndrome and DM2 can be derived, which have common pathogenic mechanisms. It is widely accepted that for prevention of these three pathologies, the main control measures are similar: a healthy diet and increased physical activity.

\section{Prevention of DM}

It is well known that dietary strategies are needed in order to prevent the onset and control of DM2, at least $80 \%$ of the population with DM2 is obese, $75 \%$ suffers hypertension and approximately $50 \%$ of DM2 patients are dislipidemic (mainly low high-density lipoprotein cholesterol (HDL) and hypertriglyceridemia). General dietary prescriptions for both obese and DM2 patients include well known diets of controlled calorie intake and predominant consumption of certain food groups like vegetables, legumes, fruits and nuts, whole-grain cereals, olive oil, wine and fish.

Risk Prediction Factors for DM2. In 2007, the International Diabetes Federation (IDF) published a consensus on DM2 prevention and defined non-modifiable and modifiable risk factors (Alberti et al., 2007). Among the first are genetic factors, age - at an older age frequency of DM2 is higher - and previous gestational diabetes (about $50 \%$ of women with a history of gestational diabetes present DM2 10 years after delivery). Other non-modifiable risk factors are low birth weight children (fetal undernutrition) and big for gestational age babies, who frequently develop obesity as adults and, consequently, diabetes. The main individual modifiable risk factor is obesity, particularly of the android type and, secondly, physical inactivity. There is uncertainty regarding the influence of certain dietary factors, such as a possible involvement of diets that are high in caloric content, saturated fatty acids and low in unsaturated fatty acids and fiber.

Physiopathology of DM2. An important fact for being able to prevent a disease is the existence of a long latency period, which allows the individual to take favorable actions. DM2 meets this requirement, as it has a slow progression and is preceded by identifiable, reversible stages. These begin with an insulin resistance (IR) state with compensatory insulin secretion sufficient to maintain normoglycemia, with hyperinsulinemia values. Subsequently, there begins a progressive fall in insulin levels, when the beta cell is no longer able to maintain hyperfunction, and as insulin secretion deficiency begins, glycemia levels increasingly rise. Initially postprandial glucose is affected, and later fasting glucose, a state designated as "prediabetes". If no measures are taken, after approximately 10 years of the IR state, clinical DM2 develops. Other physiopathological alterations in DM2 patients that are still under study are the existence of an accelerated gastric emptying and defects in the regulation of feeding, which may explain obesity of these patients. 
Search for individuals at risk for DM2. In 2007 international experts established the necessary steps for preventing DM2, based on controlling the modifiable risk factors (Alberti et al., 2007). They defined two groups, separating persons at high risk of developing DM2 from the rest of individuals. Once the former have been identified, it is necessary to measure the risk level with a score, in order to take the necessary actions. For identifying persons over 18 years with a high risk for DM2, a questionnaire is applied which should include the following factors: (android) obesity, age, family history of DM, cardiovascular and in women of gestational diabetes, and the use of diabetogenic drugs (glucocorticoids, adrenergic beta antagonists, thiazides and thyroid hormones). For a precise risk measurement, some models have been proposed, such as the non-invasive, low cost Diabetes Risk Score by Lindström (Lindström \& Tuomihleto, 2003), which does not include laboratory tests.

To date, evaluation guidelines recommended by the international organizations to establish with higher certainty a predictive score are still lacking, in order to mathematically select those individuals with higher risk for DM; in the future, this will be a very useful tool. There is no agreement regarding the search mode in children and adolescents, and the risk factors employed are those established for adults: obesity, sedentarism, family history of DM and, additionally, other factors pertaining to adolescents, such as the increase in growth hormone during puberty and presence of polycystic ovary syndrome or hyperandrogenism; in children, low birth weight and growth retardation could be important. A search mode for pregnant women at risk for DM has not been internationally defined either.

The IDF (Alberti et al., 2007) establishes that once persons at risk for DM2 have been identified, the recommendation is to determine fasting and post-load glycemias, which allow detecting cases of glucose intolerance and non-diagnosed DM. In addition, in fasting glycemias ranging between $110-125 \mathrm{mg} / \mathrm{dl}$ (impaired fasting glucose, as defined by the IFG), the recommendation is to perform an Oral Glucose Tolerance Test (OGTT) with $75 \mathrm{~g}$ glucose. Those persons with glycemia of $140-200 \mathrm{mg} / \mathrm{dl}$ are glucose intolerant (GI). Both states are designated as prediabetes and, if no pharmacological and non-pharmacological measures are received, they will develop DM2 in the short term.

\subsection{Interventions for preventing or delaying DM2}

Prevention with changes in lifestyle. Since the Malmö study, Sweden 1991, there exists evidence demonstrating that changes in lifestyle may delay or prevent DM2, in prediabetic individuals and in those at high risk of developing the disease, even if glycemias are normal. Research carried out in different countries and mainly in Caucasian subjects (the Malmö study, the Finnish Diabetes Prevention Study (DPS) and the US Diabetes Prevention Program (DPP, 2002), achieve similar results, with a decrease in the relative risk (RR) of developing DM2 of $63 \%, 58 \%$ and $58 \%$, respectively (Table 1). Studies performed in Chinese individuals, in the city of Da Qing, and in India, Indian Diabetes Prevention Program (IDPP), obtain a smaller risk reduction, of $40 \%$ and $29 \%$, respectively. The most remarkable conclusion is that for all studied ethnic groups, it is possible to prevent DM2. 


\begin{tabular}{cccccc}
\hline $\begin{array}{c}\text { STUDY } \\
\text { Country }\end{array}$ & $\mathbf{n}$ & $\begin{array}{c}\text { Years of follow- } \\
\text { up }\end{array}$ & $\begin{array}{c}\text { Average age } \\
\text { (years) }\end{array}$ & $\begin{array}{c}\text { Average BMI } \\
\mathbf{( k g / m 2 )}\end{array}$ & $\begin{array}{c}\boldsymbol{u} \text { Relative risk of } \\
\text { developing DM2 }\end{array}$ \\
\hline $\begin{array}{c}\text { DA QING } \\
\text { China }\end{array}$ & 577 & 6.0 & 45 & 25.6 & $40 \%$ \\
\hline $\begin{array}{c}\text { DPS } \\
\text { Finland }\end{array}$ & 522 & 3.2 & 55 & 31 & $58 \%$ \\
\hline $\begin{array}{l}\text { DPP } \\
\text { USA }\end{array}$ & 3234 & 2.8 & 51 & 34 & $58 \%$ \\
\hline $\begin{array}{l}\text { IDPP } \\
\text { India }\end{array}$ & 531 & 3.0 & 45.4 & 25.8 & $29 \%$ \\
\hline
\end{tabular}

Table 1. Studies on DM2 prevention with lifestyle changes

The five studies discussed have in common that the groups submitted to intervention received feeding advice for reducing weight, plans of structured physical exercise and periodic visits from the medical team for controlling compliance of the indications; on the contrary, the individuals who constituted control groups were only observed, without receiving any special indications. The results of the follow-up of DPS (Lindström et al., 2006) after the end of the three-year intervention period in subjects who had not developed diabetes are very interesting to note, as a $43 \%$ reduction of the RR of incidence of DM2 was found. The authors believe that the final success obtained in prediabetic patients is due to the fact that these individuals permanently adopted a healthy lifestyle with greater physical activity, dietary changes and weight loss.

The Da Qing study published similar results to the DPS in an observational study of up to 20 years. It showed a risk reduction of $43 \%$ in intolerant patients who were followed for up to 14 years after the end of the 6-year intervention period. The beneficial preventive effect for DM2 does not disappear at the end of the study period, but extends for many more years, probably through permanent lifestyle modifications which were internalized by the individuals.

\subsection{DM2 prevention with pharmacological measures}

The success obtained in DM2 prevention with changes in lifestyle encouraged many researchers to perform pharmacological interventions in glucose intolerant patients, especially with pharmacological measures. In Table 2 we summarize findings from several reports on the use of antidiabetic drugs, indicating the number of subjects involved, the years of intervention and the results of these programs on lowering the RR of DM2.

The DPP study (2002) in which glucose intolerant individuals received the metformin but without indications for a change in lifestyle had a much lower efficacy in preventing DM2 $(31 \%)$, than that obtained with non-pharmacological measures (58\%). Very similar achievements were shown by the group with metformin of the IDPP, with the same design and methodology as the previous work. In spite of the relatively modest results with the use of 


\begin{tabular}{ccccccc}
\hline Study & Drug & $\mathbf{n}$ & $\begin{array}{c}\text { Years of } \\
\text { follow-up }\end{array}$ & $\begin{array}{c}\text { Average age } \\
\text { (years) }\end{array}$ & $\begin{array}{c}\text { Average BMI } \\
\mathbf{( k g / m 2 )}\end{array}$ & $\begin{array}{c}\downarrow \text { Relative risk of } \\
\text { developing DM2 }\end{array}$ \\
\hline DPP & Metformin & 3234 & 2.8 & 51 & 34 & $31 \%$ \\
\hline STOP NIDDM & Acarbose & 682 & 3.3 & 55 & 31 & $25 \%$ \\
\hline IDPP & Metformin & 531 & 3.0 & 46 & 25.8 & $78 \%$ \\
\hline
\end{tabular}

Table 2. Studies on DM2 prevention with pharmacological interventions

metformin, this insulin-sensitizing medicament is the drug of choice for the pharmacological treatment of the prediabetes state, when additional high-risk factors exist. Presently, it is the drug recommended by the ADA, due to its low side effects and proven therapeutic action.

There are lesser benefits obtained with acarbose in the STOP- NIDDM study with a $25 \%$ decrease in RR, in addition to producing marked undesirable digestive side effects and premature drop-out in $31 \%$ of the subjects, due to said cause. The use of glitazones is under discussion: Troglitazone and Rosiglitazone have been withdrawn from the market due to cardiovascular risk, and the future of Pioglitazone, used in the ACT NOW study, is uncertain.

Myths or realities in DM2 prevention. Consumption of coffee is widespread throughout the world; it contains caffeine and magnesium which affect glucose metabolism; high coffee intake has been related to lesser risk of DM2 in the USA, Europe and Japan. The phytochemicals in coffee could have a positive effect in slowing down the development of DM2 (van Dam et al., 2006). In Japanese individuals, it was found that consumption of green tea reduces the risk for DM2; flavonoids from vegetables and fruit - also contained in green tea - have insulin activity and increase the action of the hormone (Iso et al., 2006).

One possibility for preventing DM2 would be the administration of vitamins E and C, which improve insulin sensitivity by inhibiting oxidative stress and inflammation, respectively. Rizzo et al. (2008), in a controlled study in older men with IFG, found that daily intake of 1000 mg of vitamin E combined with 1000 UI of vitamin C for four weeks, decreased inflammation and improved insulin action, by increasing non-oxidative glucose metabolism, glycemia, insulinemia and lipids. It has been proposed that, due to their potential anti-inflammatory and autoimmune effects, in humans calcium and vitamin D supplements would reduce the incidence of type 1 diabetes and possibly of DM2 (Danescu et al., 2009). The above findings open new prospects for the pharmacological or non-pharmacological prevention of DM2.

DM2 prevention in primary health care. The IDF in its consensus of 2007 (Alberti et al., 2007) addresses this issue and states that "intervention for delaying or preventing diabetes will cause an important reduction of its incidence, its complications and co-morbidities", and recommends controlling the modifiable risk factors: 
- - in the whole population and

- - in individuals at high risk of presenting DM2.

For prevention in the population, the IDF suggests to organize National Plans of Diabetes Prevention, adapted to the cultural conditions of each country and supported by existing organized social groups, which might start in nurseries, pre-schools and schools. Programs must have a broad coverage and must be oriented to controlling the two main modifiable risk factors, obesity and sedentarism with the purpose of therapeutic emphasis directed to changes in lifestyle.

\section{Dietary strategies for the control of diabetes}

The goals of nutritional therapy for diabetes are to prevent and treat complications such as cardiovascular disease, hypertension, nephropathy, obesity and dyslipidemia, and include achieving and maintaining safe or normal blood glucose levels, a normal lipoprotein profile, and an improvement of health through a change of food choices and increased physical activity. A healthy and balanced diet should provide enough calories for daily energy requirements, in order to reduce and/or maintain an acceptable body weight.

\subsection{Metabolic control of DM2}

It is necessary to know the degree of metabolic control in the type 2 diabetic patient in order to make the proper decisions for his/her treatment (Redsalud, 2006). It has been shown that there is a direct correlation between chronic hyperglycemia and damage to the patient's target systems and organs. The main tests for metabolic control are: glycosylated hemoglobin A1c (HbA1c) and glycemia.

HbA1c. It is currently the best control parameter of glycaemia and reports retrospectively, the glycemia average for a period of 6 to 12 weeks. Two important studies, UKPDS and DCCT, which demonstrated the relationship between metabolic control and appearance or aggravation of microangiopathic complications, employed it as an indicator of the degree of metabolic control. The goal of the treatment is to achieve $\mathrm{HbA} 1 \mathrm{c}<7 \%$, with individualized variations, the recommendation being that the $\mathrm{HbA1c}$ levels should be "the closest to normal, with the least risk of hypoglycemia for the patient". The recommended frequency of check-ups is every 3 months. Considering the heterogeneity of the elderly, goals may be variable, with $7 \%$ in a patient in good conditions, to $8.0-8.5 \%$ in a patient with limited life expectations due to comorbidities or with scarce awareness of hypoglycemias.

Glycemia. All patients should be checked with periodic laboratory glycemias or glycemias measured by capillary blood monitors in the clinic. In DM2 patients, this check-up should be carried out every three months. The goals or targets required for pre-prandial and postprandial glycemias appear in Table 3. Capillary glycemias assessed by the patient through self-monitoring are necessary only in DM2 patients on insulin treatment; the usefulness of selfmonitoring has not been demonstrated in DM2 patients with oral treatment. 


\begin{tabular}{cc}
\hline Glycosylated hemoglobin A1c & $<7 \%$ \\
\hline Pre-prandial glycemias & $70-130 \mathrm{mg} / \mathrm{dl}$ \\
\hline Post-prandial glycemias & $160-180 \mathrm{mg} / \mathrm{dl}$ \\
\hline
\end{tabular}

Table 3. Metabolic Goals

\section{Target organ compromise}

Retina. The fundus study should be performed at diagnosis of DM2. If normal, it should be repeated annually; if altered, the patient should be referred to a specialist.

Renal compromise. Assessment of plasma creatinine and calculation of glomerular filtration should be performed at diagnosis of DM2 and, if normal, should be repeated annually. Determination of proteinuria is performed at diagnosis of DM2; if negative, a microalbuminuria test is requested. Urinary albumin excretion (UAE) is quantified with the albumin/ creatinine ratio in a random urine sample. UAE values between 30 and $300 \mathrm{mg} / \mathrm{g}$ should be repeated; microalbuminuria is diagnosed when 2 out of 3 samples continue to be altered. If the UAE values are $<30 \mathrm{mg} / \mathrm{g}$, the check-up can be performed annually.

Neuropathy and arteriosclerosis of the lower limbs. The clinical examination is conducted with exploration of feet and testing of tactile and vibratory sensitivity, reflexes and peripheral pulses. This examination is conducted at the patient's diagnosis; if no alterations exist, it should be systematically repeated once a year. In this examination, it is advisable to include the orthostatism test.

Detection and prevention of cardiovascular disease (CVD). DM in general is a risk factor for CVD and DM2 is associated with other factors which form part of the pre-diabetic status and which exert negative interrelations leading to a more precocious and severe CVD. Detection and control of these factors forms part of the treatment of DM; the most important are arterial hypertension and dyslipidemia.

Arterial hypertension. Diabetic patients have a high risk of CVD starting from the prehypertension stage, which also contributes to microvascular damage. Its optimized treatment reduces micro- and macrovascular complications of DM. It is essential to guarantee detection, control and treatment of hypertension. The goals to be achieved are $<130 / 80 \mathrm{~mm} \mathrm{Hg}$ and, in nephropaths, $<125 / 75 \mathrm{~mm} \mathrm{Hg}$.

Dyslipidemia. Lipid alterations are a known risk factor for CVD and are more aterogenic in DM. The most important goal is to correct LDL cholesterol. Added to this is the so-called "aterogenic" dyslipidemia of the diabetic and pre-diabetic patient: elevated triglycerides, low HDL cholesterol and small, dense particles of LDL cholesterol. This explains why detection and treatment of lipid alterations is a main part of the check-up and follow-up of DM2. Lipid profiles should be included in the initial evaluation of DM2. If the result is normal, it should be repeated once a year. The goals to be achieved are: triglycerides $<150 \mathrm{mg} / \mathrm{dl}$, HDL $>40 \mathrm{mg} /$ $\mathrm{dl}$ in men, $>50$ in women and LDL $<100$ in primary CVD prevention and $<70$ in secondary CVD prevention. 
With the aim of achieving an optimal metabolic control and prevention of micro- and macroangiopathic complications, all patients should be encouraged to adhere to a healthy diet, low in cholesterol and saturated fatty acids and high in dietary fiber. Additionally, regular physical activity for 150 minutes per week, fractionated in 5 days, should be promoted.

\subsection{Nutritional plan for DM2}

Programmed diet (ADA, 2004) is one of the pillars of the treatment of DM2; without it, a proper metabolic control is not achieved, and the patient should permanently adhere to it. In some cases, together with exercise for some time, they both constitute sufficient total treatment for the disease.

Nutritional requirements. Nutritional requirements of persons with diabetes are similar to those of individuals without the disease. Calculation of total calories depends on the physical activity and the nutritional status of the patient. For an estimation of daily calories, the factors listed in Table 4 are used.

In overweight or obese individuals, a weight loss of $5-10 \%$ of the present weight improves blood pressure and glycemic and lipid levels. Knowing the composition of the foods, the ingredients of kitchen recipes and the amounts that should be eaten, it is possible to design the dietary plan and apply the diet for the family group.

\begin{tabular}{cccc}
\hline \multicolumn{2}{c}{ Nutritional status } & \multicolumn{2}{c}{ Physical activity } \\
\hline & Sedentary & Moderate & Intense \\
\hline Obese $\left(\mathrm{BMI}>30 \mathrm{~kg} / \mathrm{m}^{2}\right)$ & $20-25$ & 30 & 35 \\
\hline $\begin{array}{c}\text { Overweight }(\mathrm{BMI}>25-29.9 \\
\left.\mathrm{kg} / \mathrm{m}^{2}\right)\end{array}$ & $26-29$ & 28 & 30 \\
\hline $\begin{array}{c}\text { Normal }\left(\mathrm{BMI} 20-24-9 \mathrm{~kg} / \mathrm{m}^{2}\right) \\
\text { Lean }\left(\mathrm{BMl}<20 \mathrm{~kg} / \mathrm{m}^{2}\right)\end{array}$ & 30 & 35 & 40 \\
\hline
\end{tabular}

Table 4. Caloric recommendations for adults. Calories/ kg of acceptable body weight

Carbohydrates $(\mathrm{CH})$. The percentage of carbohydrate calories varies, it is individual and based on eating habits, targeted glycemia and lipids. The recommended range is between $55 \%$ and $60 \%$ of the total daily calories. Restriction of carbohydrates to less than 130 grams/day is not recommended, since the brain and the nervous system have an absolute requirement for glucose as a source of energy. In the dietary plan, it is advisable to leave out simple sugars (honey, panela, molasses, sugar) or lower them in well-controlled patients to $<5 \mathrm{~g}$ per day.

Lipids. In the type 2 diabetic patient, elevations in very low density lipoprotein (VLDL) concentrations, reductions in high density lipoprotein (HDL) concentrations and elevations in the low density fraction (LDL) may be found. Type 2 diabetics may have an altered lipid profile, notwithstanding a good glycemic control. 
The main goal in lipid intake is to reduce consumption of saturated fatty acids and trans fatty acids. Lipids should account for less than $30 \%$ of the total daily caloric intake. Foods containing monounsaturated and polyunsaturated fatty acids should be preferred in order to comply with the recommendations. Less than $7 \%$ of the calories provided by saturated (SFA) and trans fatty acids is recommended. SFAs are the main source of formation of LDL cholesterol and are found in fatty meats, dairy products and eggs. Trans fatty acids are produced by hydrogenating vegetable or fish oils in order to achieve a more solid texture.

Monounsaturated fatty acids (MFA) are recommended in a proportion of $13 \%$, especially in the cis configuration, as found in olive oil, sesame, nuts and peanuts. MFAs may be beneficial in the control of blood lipids and of cardiovascular diseases. It is indicated that less than $10 \%$ of the calories should be provided by polyunsaturated fatty acids (PFA), which are found in vegetable oils such as corn, sunflower, safflower and soybean oils. Omega 3 eicosapentenoic and docosahexenoic fatty acids (EPA and DHA) present in fish have a beneficial effect on triglycerides. It is recommended not to exceed an intake of $200 \mathrm{mg}$ of cholesterol per day. Consumption of fish or fish oil containing omega-3 polyunsaturated fatty acids (PUFAs) reduce the risk of coronary heart disease, decrease mild hypertension, prevent certain cardiac arrhythmia and lower the incidence of diabetes. Omega-3 PUFAs play a vital role in the development and function of the nervous system and photoreception. Dietary lipids should be lowered if weight loss is desired; if this dietary scheme is maintained for a long term, it contributes to weight loss and to ameliorate dyslipidemia.

The recommended lipid composition in the diet of a type 2 diabetic patient is set forth in Table 5 .

\begin{tabular}{l}
$<7 \%$ of the calories: saturated (SFA) and trans fatty acids \\
$<10 \%$ of the calories: polyunsaturated fatty acids (PFA) \\
$13 \%$ of the calories: monounsaturated fatty acids (MFA) \\
\hline Omega 3 fatty acids (cis configuration) $0.15 \mathrm{~g} /$ day
\end{tabular}

Table 5. Contribution of dietary fatty acids in a DM2 patient

Proteins. Proteins should account for $15-20 \%$ of total caloric intake, $0.8-1.0 \mathrm{~g} / \mathrm{Kg}$ of body weight; dietary protein intake must be balanced and rich in essential amino acids. According to the dietary guidelines, the intake of protein in the usual range does not appear to be associated with the development of diabetic nephropathy, although it is advisable to avoid intakes over $20 \%$ of the total daily energy.

The Recommended Dietary Allowance (RDA) indications for the general population of $0.8 \mathrm{~g}$ of proteins of high biological value, per $\mathrm{kg}$ of weight per day in the adult, are still followed. Proteins of high biological value provide all of the essential amino acids and in the amounts required by the body; they are the proteins of animal origin, all types of meat (beef, pork, fish, etc), milk and eggs. When the drop in glomerular filtration is initiated, proteins should not be 
restricted beyond $0.6 \mathrm{~g} / \mathrm{kg} /$ day in order not to cause malnutrition. In the highly uncompensated type 2 diabetic patient, there could occasionally be a loss of body proteins.

Glycemic index. It is established as the incremental area under the glycemia curve produced by the intake of a standard amount of available CHs of a food (usually $50 \mathrm{~g}$ ), in relation to the same amount of carbohydrates from a standard source (glucose or white bread). Recommendation is to prefer foods with low glycemic index (legumes, green vegetables).

Dietary fiber. Soluble fiber of vegetables, legumes and fruit (pectin) helps to control not only glycemia but also lipids, and should be accompanied by an increase in water intake. Fibers also help satiety. Processed foods tend to decrease the amount of available fiber. Complex carbohydrates, which also have a high percentage of soluble dietary fiber, are present in legumes, vegetables and fruits, and should be included in a healthy diet.

Sweeteners. Non-caloric sweeteners such as saccharin, aspartame, acesulfame K, sucralose, estevioside are accepted as safe, as long as their accepted daily intake is observed.

Sodium. Individuals differ in sensitivity to sodium intake, related to arterial pressure. Sodium recommendation is the same as for the general population: not more than $2.4 \mathrm{~g} /$ day, equivalent to $6.0 \mathrm{~g} /$ day of $\mathrm{NaCl}$.

Alcohol. The same recommendations as for the general population are valid. Never to drink on an empty stomach and to prefer red wine due to its phenol (antioxidant) content; the maximal recommended portion is not more than 2 cups per day in men and not more than one in women. $100 \mathrm{ml}$ of wine contain 9-13 g of alcohol, and one gram of alcohol provides 7 calories. Alcohol abstention is recommended during pregnancy, in hypertriglyceridemia, pancreatitis, arterial hypertension or neuropathy. If the individual with diabetes is abstemious, he/she will be advised not to initiate this practice. Fruit and vegetable intake provides a suitable amount of antioxidants.

Vitamins and Minerals. A suitable dietary intake provides vitamins and minerals in sufficient amounts and no supplementation is necessary. However, there are some exceptions: individuals on chronic hypocaloric diets, which should be supplemented with iron and B-Complex vitamins; iron, folate and calcium supplements are recommended to pregnant women; calcium supplement is recommended to women with osteoporosis; and undernourished subjects are advised to take supplements appropriate to the deficiency they present. Vitamin supplementation with antioxidants (vitamins E, C and A) is not advisable as a routine, due to lack of evidence and safety and long-term safety. Chromium, Selenium, Zinc and Manganese are contained in the usual diet, while supplements are recommended only when patients have a deficiency.

The nutritional plan detailed for DM2 is also recommended for individuals who are obese, insulin-resistant, pre-diabetic and carriers of metabolic syndrome, in order to prevent them from developing DM2.

To summarize, one of the key measures to achieve control targets in patients with diabetes is a diet plan, as there is ample evidence that the goals are not achieved if patients fail nonpharmacologic measures, diet and physical activity. 


\section{Nutrition and social behavior}

The drastic and long-term changes in eating habits that are needed in order to prevent and control these diseases require a highly motivated and educated population with the economic means and a strong personal commitment to follow and maintain these indications. Changing dietary habits may be a very difficult task to accomplish and constant support is needed from health professionals who should develop individual therapeutic programs for each patient according to their personal lifestyle requirements, tastes or cultural backgrounds.

Why is it so difficult to achieve this change to healthy eating habits? In the first place it is important to understand that eating is not an isolated behavior, as in all societies it is part of the cultural context that defines what is considered edible or appropriate (or not) for each member of any particular group. Contreras \& Grace (2005) suggest the presence of a food culture that accompanies any food system, which is defined as follows: "the set of representations, beliefs, knowledge and practices inherited and / or learned that are associated to food and that are shared by individuals of a given culture or a social group within a culture". In addition to keeping alive and avoiding overt hunger, food all over the world and in all possible settings is also used to keep and classify human relationships, indicate social status, enhance political, business and economic relations, provide praise and punishment, prevent and treat physical and mental disease, to indicate religious adherence or membership, to evidence emotions and even morals. As illustrated above, the underlying development of attitudes, from infancy to old age, in pregnancy and disease, for adolescents and adults influence food behavior and are determined by culture, economics, food technology, politics, the family and recently, by mass media. Consequently, to achieve significant impact, education in nutrition and dietetics also requires knowledge of the socio-cultural aspects of eating as well as its basic scientific aspects, and the professional providing nutrition services and education in the community needs an understanding of these facts (Bass et al., 1979). Briefly, food and food consumption patterns underlie the social fabric in all societies and hence its cultural importance cannot be underestimated.

Second, the social act of eating has changed along the millennia of humankind; therefore it is of use to put the nutritional transitions into a historical context. From a long history of omnivorism of hunter-gatherers (mainly plants supplemented with occasional meat), that shaped our physiology to the present day, humans have moved through several important transitions in their nutrition. Omnivorism probably required cooperation between members of a species that is rather ill-equipped compared to other predators, possibly setting the stage for social patterns for providing and distributing food (Aguirre, 2010). These conditions selected among our ancestors those individuals endowed with genes that permitted the accumulation of calories in times of surplus, and survive in times of low access to food or starvation (Aguirre, 2010).

Later, approximately 15.000 to 10.000 B.P. a major nutritional transition occurs as an adaptation to the higher temperatures of the postglacial era (and the associated decrease of large prey for hunting) which leads to the development of agriculture (Smith \& Munro, 2009). The introduction of staple food lead to the establishment of villages and an increase of the human 
population and a shift to meals based on cereals (i.e. carbohydrates) and a decrease of protein intake. As a result, on a global scale humans on average became approximately $20 \mathrm{~cm}$ shorter with a decreased life expectancy due to chronic malnutrition of micronutrients. Also, specific diseases related to agricultural labor and food processing appeared, such as arthritis, skeleton deformities and dental abrasion, particularly in women (Aguirre, 2010). The establishment of sedentary societies also set the stage for classes with differential foods, creating small well-fed upper classes with large, fat bodies and a majority of the population with the short and lean phenotype of poverty, fed a low variety of (or a single) cereal or tuber staple (Aguirre, 2010).

The next nutritional transition comes about with the industrial revolution, which initially causes a severe drop in the quality of life for the large majority of the population in the western world. The new technologies completely change the methods of producing, distributing and consuming foods. The modern relocation of edible plants throughout the world introduces the concept of plantations, and worldwide distribution of some edible products such as sugar, coffee, tea and potatoes, among others (Aguirre, 2010). The economic powers involved provoke colonial wars, and in the long run, create the industrial food production with large scale food transportation and storage, a panoply of additives (flavorings or dyes) and stabilizers, and the disappearance of seasonality of fruits and vegetables and local food production, that characterizes the modern food production of in our days.

The genetic makeup of our species has not changed at the pace of these nutritional transitions, so we are now faced with a large population, living in a largely industrial-urban setting that still maintains those biological features that constituted an advantage for survival in the past. Although there are 870 millions of people suffering hunger today (http://faostat3.fao.org/ home/index.html), for the first time in human history, a large segment of the population has access to a large supply of calories in the form of carbohydrates and fats, albeit, not to a healthy diet with low fat proteins and micronutrients. Finally, this nutritional transition has happened so fast that the shift from undernourishment to obesity has occurred within just two generations. Indeed, in developing countries, it is not uncommon that an individual, who experienced undernourishment during childhood, is today an obese adult with overweight children. Therefore, in a completely different context, our genetic makeup in an era of surplus calories and changing lifestyles provides the basis for the worldwide epidemic of obesity and diabetes.

As pointed out at the beginning of this section, a highly motivated and educated population with the economic means and a strong personal commitment to follow and maintain the indications is needed to revert this situation. Low levels of education of mothers also tend to result in children that spent more time watching TV and eat a higher amount of fat and calorie-rich snacks and "junk food", highlighting the importance of enhancing school-based and community-based actions to promote healthy eating and physical activity addressed to children and young people (Aranceta et al., 2003). Also, in the face of increasing food prices, many people with lower incomes do not have a realistic possibility of choosing an appropriate diet.

Since the general population in most developing countries such as Chile, is not able to keep these recommendations in the long term for any of the above reasons, several kinds of dietary interventions have been proposed. These include low calorie foods and foods containing high- 
quality ingredients such as peptides derived from fish. Some of these strategies include the replacement of red meat and its derivatives by fish and white meat, the reduction of the total fat intake (saturated fat) and replacement by the use of other oils (such as olive oil) and a higher intake of dietary fiber, replacing white bread with whole grain bread. Here we propose to include marine-derived proteins, specifically fish-derived protein hydrolysates as high biological value proteins of low cost as dietary supplements or ingredients, as a means of preventing obesity and diabetes.

\section{Marine-derived protein}

\subsection{General aspects}

Seafood and fish are sources of high biological value proteins, unsaturated essential fatty acids, vitamins and antioxidants, minerals or trace metals and physiological beneficial amino acids and peptides. Additional components in seafood may be of importance for the development of life style diseases like coronary heart disease. There is ample literature on marine-derived peptides (salmon and other fish) with biological activity against obesity, DM2 and cardiovascular disease, which leads to the proposal of using protein concentrates produced by enzymatic hydrolysis from co-products of the fish industry that may contain these peptides. Several research groups, including ourselves, are concentrated on generating an application or nutraceutical biological functionality of these products.

Different strategies have been studied for recovering protein from the marine products industry, as for example fish protein hydrolysates (FPHs, by its acronym in English). These treatments are obtained by solubilizing the raw protein, via peptide bond breaks which release smaller peptides and free amino acids, that can then be separated and recovered. In this type of application protein recovery by enzymatic hydrolysis is chosen, because this treatment gives rise to a final product of higher digestibility than untreated protein (Aurrekoetxea \& Perera, 2002).

Several studies reveal that proteins derived from hydrolysis of marine products are of high nutritional value, rich in essential amino acids and even similar to proteins from beef (Kristinsson \& Rasco, 2000). It has also been observed that hydrolysates, treated with commercial enzymes, exhibit functional characteristics (solubility, fat absorption, water absorption and stability of the emulsion) consistent with potential uses as emulsifying agents, and absorbing agents, being potentially competitive with existing protein hydrolysates, such as dairy products and vegetables, which are currently available on the market (Sathivel et al., 2005).

Enzymatic digestion of proteins of marine origin results in FPH. Among the problems of obtaining the FPH, is the extreme susceptibility to oxidation of fats, and the presence of these oil residues which generate unpleasant odors and flavors from an organoleptic viewpoint (Hoyle \& Merritt, 1994). 
Protein hydrolysates also have non-food applications such as power supply for the growth of microorganisms such as yeast hydrolysates or casein. They can also be used eventually as plant fertilizers and also used in cosmetics for hair treatment (due to its suggested effect of strengthening of hair) (Vioque \& Millan, 2005).

Enzymatic hydrolysis has clear advantages over traditional acid or alkaline chemical hydrolysis, for the following reasons (Guadix et al., 2000):

- Selectivity. Enzymes are specific for a particular type of chemical bond, and the appearance of degradation products is not common. In contrast, the low selectivity of the acidic and basic treatments inevitably leads to the appearance of degradation products that are difficult to control and may be toxic.

- Moderate temperature and $\mathrm{pH}$ conditions. Enzymatic hydrolysis generally proceeds in the range of 40 to $60^{\circ} \mathrm{C}$ and $\mathrm{pH}$ of 4.0 to 8.0 .

- No foreign substances are added. Chemical hydrolysis processes are carried out with strong acids or bases, which are then neutralized, thereby significantly raising the salt content of the product.

- Maintain the nutritional value. There is no degradation of the components, while alkaline hydrolysis destroys the amino acids arginine and cysteine, and acid hydrolysis removes tryptophan and deaminates serine and threonine.

As a result of biological hydrolysis, high-protein, low-fat FPHs can be obtained, which are completely soluble products (Aurrekoetxea \& Perera, 2002).

\subsection{Physiological effects of marine-derived peptides and aminoacids}

Potent peptides with high anti-hypertensive activities (inhibitors of angiotensin I-converting enzyme or ACE) and peptides which may modulate central neuropeptide levels have been isolated from fish hydrolysates (Yoshikawa et al., 2000; Sorensen et al., 2004). Marine low molecular weight components antioxidants (tocopherols, CoQ10, selenium, taurine) have attracted special attention due to their possible prevention of low-density lipoprotein (LDL) oxidation. Fish proteins have also been shown to inhibit LDL oxidation in rat models (Kondo et al., 2000).

It has been documented that peptides obtained from fish muscle digests possess potent inhibitory activity against ACE and antihypertensive properties (Galardy et al., 1984; Kohama et al., 1996). For assessment of relative antihypertensive activities of two peptides (LKPNM and LKP) derived from bonito fish to that of captopril, administered orally to SHR rats to monitor time-course changes of blood pressures (Fujita \& Yoshikawa, 1999). Both LKPNM and captopril showed maximal decrease of blood pressure $4 \mathrm{~h}$ after oral administration and their efficacies lasted until $6 \mathrm{~h}$ post-administration. In sharp contrast, however, maximal reduction of blood pressure occurred as early as $2 \mathrm{~h}$ after administration of LKP. When compared on molar basis, antihypertensive activities of LKPNM and LKP accounted for $66 \%$ and $91 \%$ relative to that of captopril, respectively, whereas in vitro ACE-inhibitory activities of LKPNM and LKP were very low compared with that of captopril. It is of interest to note that both of 
these peptides exerted remarkably higher antihypertensive activities in vivo despite weaker in vitro ACE-inhibitory effects, using captopril as the reference drug (Fujita \& Yoshikawa, 1999). Such peptides may be regarded as healthy components (through endogenous metabolism) of fish muscles and additionally may be produced as ingredients or diet supplements.

Amino acids (taurine) and peptides (ACE -inhibitors) are beneficial components from seafood and hence components in possible ingredients in functional foods. Seafood contains high levels of the amino acid taurine and the consumption of seafood has been shown to increase its serum concentration (Laidlaw et al., 1990; Kim et al., 1996). The suggestion of a possible association between fish intake and reduced cardiovascular risk through the beneficial effects of proteins and taurine, in addition to omega n-3 fatty acids, has been put forward (Mizushima et al., 1997; Yamori et al., 1994).

In humans, taurine is regarded to be a conditionally essential amino acid, as its physiological concentration can be partly regulated endogenously. Taurine is known to have several positive effects on the cardiovascular system, as described in a broad review by Niittynen et al. (1999). Firstly, taurine has antioxidant activity. This may reduce the production of proinflammatory products. Secondly, taurine has been shown to lower blood pressure in borderline hypertensive patients. It has also been reported that taurine can improve cardiac performance, reduce blood cholesterol values and suppress platelet aggregation (Niittynen et al., 1999). A protective role from both taurine and ACE inhibitors has been found in age-related progressive renal sclerosis in 24 and 30 months old rats. Taking into account the antioxidant properties of taurine, these data suggest a role for ROS in age-related progressive renal fibrosis, perhaps through interactions with de TGF- $\beta 1$ pathway (Iglesias-de la Cruz et al., 2000). Although commonly used as a dietary supplement in the Far East, the potential advantages of dietary taurine consumption /supplementation have not been recognized in the Western World (Stapleton et al., 1998).

Frozen-preserved commercial diets have been shown to maintain plasma taurine concentration, whereas the heat-processed diets do not (Kim et al., 1996; Dragnes et al., 2009). On the other hand, a significant correlation was found between the results of the biological assessment of the nutritional value of processed protein and of taurine content in the liver and urine of rats (Lipka et al., 1993).

\subsection{Improvement of FPHs through the use of enzymes}

The functional properties of FPHs can be improved by the use of specific enzymes and by the choice of the hydrolysis conditions, such as time, temperature and $\mathrm{pH}$. In this way partial hydrolysis can be achieved. There are a number of different commercial proteolytic enzymes that can be used to produce hydrolysates (Liceaga-Gesualdo \& Li-Chan, 1999).

The addition of exogenous enzymes to the hydrolytic process makes it more controllable and reproducible. Consequently, different commercial proteolytic enzymes have been tested on a variety of marine substrates. The preferred commercial enzymes are proteases of bacterial origin, such as Alcalase, Neutrase, Protamex (bacterial proteases trade names), but also the 
plant proteases such as papain (commercially called Corolase L-10), present good yields in hydrolytic processes (Aspmo, 2005).

All enzymes used for hydrolyzing proteins of marine origin have to be of food grade, and if they are of bacterial origin, the production organism needs to be non-pathogenic. The choice of enzyme is usually determined by a combination of effectiveness and economy (Kristinsson \& Rasco, 2000). Major factors in the choice of enzymes are the organoleptic and functional characteristics of the final food product.

\subsection{Organoleptic properties of the hydrolysate}

Although enzymatic hydrolysis of marine proteins produces peptides with desirable functional properties, it has the disadvantage of generating bitterness. This is a common problem with FPHs, and is the main reason for its low acceptance as a food ingredient. The mechanism for the development of bitterness is not yet clear, but it is widely accepted that the hydrophobic amino acids of the peptides are the main factor. The hydrolysis of proteins results in the exposure of internal hydrophobic peptides which are able to interact rapidly with the taste buds, resulting in the detection of bitter taste. However, extensive hydrolysis to produce free amino acids reduces the bitterness of peptides, because hydrophobic peptides are much bitterer compared with a blend of free amino acids, which are though, undesirable from a functional point of view. A strict control of the degree of hydrolysis in any experimental system is therefore desirable to prevent the development of bitter taste and also for the retention of functional properties (Kristinsson \& Rasco, 2000; Liaset et al., 2003; Benitez et al., 2008).

Proteases have different specificity for the amino acids they cut, so the choice of the most appropriate enzyme or mixture of enzymes depends on the raw material subjected to hydrolysis, and may affect the degree of bitterness. For instance, enzymes with a high preference for hydrophobic amino acids, such as Alcalase, are often preferred and may yield products with low bitterness. The use of exopeptidases, instead of endoproteases, may also be useful in reducing the bitterness of the FPH, particularly if exopeptidases separating hydrophobic amino acids from bitter peptides are used. However, for an enzyme preparation to be effective in protein hydrolysis, both exopeptidases and endopeptidases are required. Many studies have shown that preparations containing proteolytic exopeptidases and endopeptidases produce less bitter peptides than single proteases. The addition of exopeptidases to the process often eliminates the bitter taste of the hydrolysates (FitzGerald \& O'Cuinn, 2006).

The ideal method for the quantification of the bitterness of a hydrolysate is the use of a sensory evaluation panel. This is a time-consuming activity and requires an appropriate number of panelists trained to detect such bitterness, in order to obtain statistically relevant data (FitzGerald \& O`Cuinn, 2006; Seo et al., 2008).

\subsection{Use of antioxidants in the hydrolysis}

Oils and fatty products generally undergo oxidation during production and storage. A major problem of the preparation of FPHs is their extreme susceptibility to such oxidation fats (Hoyle \& Merritt, 1994). This oxidation process in food causes a sequence of unfavorable changes, 
consisting mainly in deterioration of the sensory properties of the product such as rancidity, changes in color and texture and reduced nutritional value. It also increases health risks, along with economic losses and may even influence the development of bitter flavors (Palić \& Lucan, 1995; Valenzuela et al., 2003; Gramza \& Korczak, 2005; Mendis et al., 2005; Dong et al., 2008).

Marine oils are rich in polyunsaturated fatty acids (PUFAs), especially of the $\omega 3$ family such as EPA and DHA, however diets containing these oils are more susceptible to oxidation than those containing other types of oils, because of their high concentrations of long chain PUFAs; the aforementioned examples are DHA and EPA (Gonzalez et al., 1992; Fritsche \& Johnston, 1988; Wanasundara \& Shahidi, 1998).

PUFA degradation via chain reaction mechanisms of free radicals, results in changes of smell and taste (rancidity) of edible oils and oil-containing foods. The chemical reactions involved in oxidative processes require low activation energy, and do not significantly change their ranges at low storage temperatures; therefore, it is necessary to delay the onset of oxidation of the marine oil to maintain its flavor and odor (Wanasundara \& Shahidi, 1998; Ahn et al., 2007).

\subsection{Antioxidants}

Antioxidants, natural and synthetic, are commonly used by the food processing industry, to prolong the storage stability of food. The main commonly used synthetic antioxidants are butylhydroxybutilanisol, butylated hydroxytoluene, propyl gallate and tertiary butylhydroquinone (BHA, BHT, PG and TBHQ, respectively, for their acronyms in English). Natural antioxidants such as tocopherols, rosemary extracts (Herbalox, commercial product) and ascorbic acid are often preferred (Gramza \& Korczak, 2005; Yu et al., 2006).

\subsection{Preliminary trials}

Preliminary tests of the organoleptic properties of enzymatic hydrolysis of marine proteins with different commercial enzymes, among them Alcalase, and Colorasa Protamex (papain) and different commercial antioxidants, showed greater acceptability of the process than with the protease Colorasa L-10. These results were observed after organoleptic analysis of the obtained products in trials with healthy volunteers (unpublished results).

While the use of antioxidants improves the stability of the oil fraction obtained together with the protein fraction, products derived from an antioxidant-free process were more acceptable (organoleptic properties) than those obtained in a process in which antioxidants were are used, although the oil fraction underwent faster oxidation (unpublished results). These results suggest that the addition of antioxidants is not of vital importance in the course of an organoleptically suitable product with a high proportion of proteins of marine origin.

\section{Concluding comments}

In this chapter we present general data concerning the worldwide epidemic of obesity and diabetes and discuss the present day goals of metabolic control of affected patients. The current 
strategies used for prevention of the development of prediabetes and diabetes are discussed and a brief up-to-date review of summarizes the dietary strategies for the nutrition of diabetic patients.

In the following section we briefly describe the main impact of historic nutritional transitions and illustrate how the social impact of present day food production, distribution and consumption is exacting a high toll on public health (and also on the environment). To amend this general pattern, large changes of society on a global scale are needed. Since these do not seem realistic in the short term, alternative strategies to address particular problems are suggested, such as nutritional intervention with high quality nutrients of low economic value such as supplementing foods with marine-derived protein hydrolysates.

A discussion of the different procedures for obtaining fish derived protein hydrolysates is presented, centered on the advantages of enzymatic hydrolysis, with respect to chemical procedures. The use of antioxidants during the hydrolysis process is also analyzed and not found to be essential for obtaining a high quality product. The organoleptic properties of hydrolysates obtained by our group have proven acceptable in trials to healthy volunteers. In addition, fish derived protein hydrolysates may contain peptides and/or aminoacids which may bear additional beneficial effect.

To summarize, healthy dietary habits are part of the necessary lifestyle to prevent obesity and DM2; an increased intake of marine products is also recommended as a means to control risk factors of cardiovascular disease of DM2 patients.

\section{Author details}

Daniela Seelenfreund ${ }^{1 *}$, Pilar Durruty ${ }^{2}$, Nicolas Palou ${ }^{1}$, Sergio Lobos $^{1}$ and Rodrigo González ${ }^{1}$

1 Department of Biochemistry, Faculty of Chemical and Pharmaceutical Sciences, University of Chile, Providencia, Santiago, Chile

2 Diabetes Unit, Department of Medicine, Faculty of Medicine, University of Chile, Santiago, Chile

\section{References}

[1] Aguirre, P. (2010). Ricos flacos y gordos pobres: la alimentación en crisis. 1rst Ed. Capital Intelectual S.A., Buenos Aires, Argentina.

[2] Ahn, J, Grün, I, \& Mustapha, A. (2007). Effects of plant extracts on microbial growth, color change, and lipid oxidation in cooked beef. Food Microbiol, , 24, 7-14. 
[3] Alberti KGMZimmet P, Shaw J, (2007). International Diabetes Federation: a consensus on Type 2 diabetes prevention. Diabetic Medicine, , 24, 451-463.

[4] American Diabetes Association(2004). Nutrition Principles and Recommendations in Diabetes.. oi: 10.2337/diacare.27.2007.S36 Diabetes Care January 2004 no. suppl 1 s36., 27

[5] Aranceta, J, Pérez-rodrigo, C, \& Ribas, L. Serra-Majem, Ll, (2003). Sociodemographic and lifestyle determinants of food patterns in Spanish children and adolescents: the enKid study. Eur J Clin Nutr, 57: Suppl 1, SS44., 40.

[6] Aspmo, S. (2005). Enzymatic hydrolysis of Atlantic cod (Gadusmorhua L.) viscera. Process Biochem, , 40, 1957-1966.

[7] Aurrekoetxea, G, \& Pereira, M. (2002). Aprovechamiento de recursos pesqueros infrautilizados para la obtención de alimentos mejorados para el cultivo de peces. Boletín del Instituto Español de Oceanografía, , 18, 87-93.

[8] Bass, M. A, Wakefield, L, \& Kolasa, K. (1979). Community nutrition and individual food behavior. Publisher Burgess Pub. Co., Minneapolis (USA).

[9] Benítez, R, Ibarz, A, \& Pagan, J. (2008). Hidrolizados de proteína: procesos y aplicaciones. Acta Bioquímica Clínica Latinoamérica, , 42, 227-237.

[10] Contreras, J, \& Gracia, M. (2005). Alimentación y Cultura: perspectivas antropológicas. Editorial Ariel. Barcelona, Spain. (Ibid. 2005: 96).

[11] Danescu, L. G, Levy, S, \& Levy, J. and diabetes mellitus. Endocrine, , 35, 11-17.

[12] Diabetes Prevention Program Research Group(2002). Reduction in the incidence of type 2 diabetes with lifestyle intervention or metformin. N Engl J Med, , 346, 393-403.

[13] Dragnes, B. T, Larsen, R, Ernstsen, M. H, Mæhre, H, \& Elvevoll, E. O. (2009). Impact of processing on the taurine content in processed seafood and their corresponding unprocessed raw materials. Int J Food Sci Nutr, , 60(2), 143-152.

[14] ENSMinisterio de Salud Chile. Encuesta Nacional, htpp:www.minsal.cl/portal/url/ page/minsalcl/g_home/home.html, 2009-2010.

[15] FitzGerald RO'Cuinn G, (2006). Enzymatic debittering of food protein hydrolysates. Biotechnol Adv, , 24, 234-237.

[16] Fritsche, K, \& Johnston, P. (1988). Rapid autoxidation of fish oil in diets without added antioxidants. J Nutrition, , 118-425.

[17] Fujita, H, \& Yoshikawa, M. (1999). LKPNM: a prodrug-type ACE-inhibitory peptide derived from fish protein. Immunopharmacology, 44( 1-2): 123-127.

[18] Galardy, R, Podhasky, P, \& Olson, K. R. (1984). Angiotensin-converting enzyme activity in tissues of the rainbow trout. J Exp Zool, , 230(1), 155-158. 
[19] González-torres, M, Betancourt-rule, M, \& Ortiz, R. (2000). Daño oxidativo y antioxidantes. Bioquímica, , 25, 3-9.

[20] Gramza, A, \& Korczak, J. (2005). Tea constituents (Camellia sinensis L.) as antioxidants in lipid systems. Trends in Food Science \& Technology, , 16, 351-358.

[21] Guadix, A, Guadix, E, Páez-dueñas, M, González-tello, P, \& Camacho, F. (2000). Procesos tecnológicos y métodos de control en la hidrólisis de proteínas. Ars Pharmaceutica, , 40, 79-89.

[22] Hoyle, N. T, \& Merritt, J. H. (1994). Quality of Fish Protein Hydrolysates from Herring (Clupeaharengus). J Food Sci, , 59, 76-80.

[23] Iglesias de la Cruz CRuiz-Torres P, García del Moral R, Rodríguez-Puyol M, Rodríguez-Puyol D, (2000). Age-related progressive renal fibrosis in rats and its prevention with ACE inhibitors and taurine. Am J Physiol Renal Physiol, 278: FF129., 122.

[24] Iso, H, Date, C, Wakai, K, Fukui, M, \& Tamakoshi, A. and the JACC Study Group, (2006). The relationship between green tea intake and type 2 diabetes in Japanese adults. Ann Inter Med, , 144, 554-562.

[25] Kim, S. W, Rogers, Q. R, \& Morris, J. G. (1996). Maillard reaction products in purified diets induce taurine depletion in cats which is reversed by antibiotics. J Nutr, , 126(1), 195-201.

[26] Kohama, Y, Kuroda, T, Itoh, S, \& Mimura, T. (1996). Tuna muscle peptide, PTHIKWGD, inhibits leukocyte-mediated injury and leukocyte adhesion to cultured endothelial cells. Biol Pharm Bull, , 19(1), 139-41.

[27] Kondo, K, Iwamoto, T, Hooda, K, Kamiyama, M, Hirano, R, Kidou, T, Matsumoto, A, Watanabe, S, \& Itakura, H. (2000). Inhibition of low-density lipoprotein oxidation by fish protein and antioxidants. XIIth International Symposium on Atherosclerosis, Abstract W12: 113. June Stockholm, Sweden.(TuP18), 25-29.

[28] Kristinsson, H. G, \& Rasco, B. A. (2000). Fish protein hydrolysates: production, biochemical, and functional properties, Crit Rev Food Sci Nutr, , 40, 43-81.

[29] Liaset, B, Julshamn, K, \& Espe, M. (2003). Chemical composition and theoretical nutritional evaluation of the produced fractions from enzyme hydrolysis of salmon frames with Protamex. Process Biochemistry, , 38, 1747-1759.

[30] Liceaga-gesualdo, A, \& Li-chan, E. (1999). Functional properties of fish protein hydrolysate from herring," J Food Sci, , 64-1000.

[31] Lindström, J, Ilanne-parikka, P, Peltonen, M, Aunola, S, Eriksson, J, \& Hemio, K. (2006). Sustained reduction in the incidence of type 2 diabetes by lifestyle intervention: follow-up of the Finnish Diabetes Prevention Study. Lancet, , 368, 1673-1679.

[32] Lindström, J, \& Tuomilehto, J. (2003). The Diabetes Risk Score. A practical tool to predict type 2 diabetes risk. Diabetes Care, , 26, 725-731. 
[33] Mendis, E, Rajapakse, N, \& Kim, S. (2005). Antioxidant properties of a radical-scavenging peptide purified from enzymatically prepared fish skin gelatin hydrolysate. J Agric Food Chem, , 53, 581-587.

[34] Nittynen, L, Nurminen, M. L, Korpela, R, \& Vapaatalo, H. (1999). Role of arginine, taurine and homocysteine in cardiovascular diseases. Ann Med, , 31(5), 318-326.

[35] Palic, A, \& Lucan, Ž. (1995). Antioxidative Effect of 'Herbalox' on Edible Oils. Lipid/ Fett, , 97, 379-381.

[36] Redsalud(2006). Www.redsalud.gov.cl/archivos/guiasges/diabetesGES.pdf http:// www.fao.org/docrep/016/i3027e/i3027e03.pdf

[37] Rizzo, M. R, Abbatecola, A. M, Barbieri, M, et al. (2008). Evidence for anti-inflammatory effects of combined administration of vitamin $\mathrm{E}$ and $\mathrm{C}$ in older persons with impaired fasting glucose: impact on insulin action. J Am Coll Nutr, , 27, 505-11.

[38] Sathivel, S, \& Smiley, S. (2005). Functional and nutritional properties of red salmon enzymatic hydrolysates. Journal of Food, , 70, 401-406.

[39] Smith, A, \& Munro, N. D. (2009). A holistic approach to examining ancient agriculture. Curr Anthropol, , 50, 925-936.

[40] Valenzuela, A, Sanhueza, J, \& Nieto, S. (2003). Natural antioxidants in functional foods: from food safety to health benefits. Grasas y aceites, , 104, 754-760.

[41] Van Dam, R, Willett, W, Manson, J, \& Hu, F. (2006). Coffee, caffeine, and risk of type 2 diabetes. Diabetes Care, , 29, 398-403.

[42] Vioque, J, \& Millán, F. (2005). Los hidrolizados proteicos en alimentación: Suplementos alimenticios de gran calidad funcional y nutricional. Agro CSIC, , 2-8.

[43] Wanasundara, U, \& Shahidi, F. (1998). Antioxidant and pro-oxidant activity of green tea extracts in marine oils. Food Chemistry, , 63, 335-342.

[44] World Health Organization Prevalence of Diabetes(2010). htpp://www.who:int/ diabetes/facts/world:figures/en/indexx2.html

[45] Yu, H-H, Liu, X, Xing, R, Liu, S, Guo, Z, Wang, P, Li, C, \& Li, P. (2006). In vitro determination of antioxidant activity of proteins from jellyfish. Food Chemistry, , 95, 123-130. 